\title{
Chirality-enabled unidirectional light emission and nanoparticle detection in parity-time-symmetric microcavity
}

\author{
Weihua Wang, ${ }^{1}$ Shuai Liu, ${ }^{2}$ Zhiyuan Gu, ${ }^{1,{ }^{*}}$ and Yue Wang $\oplus^{1, \dagger}$ \\ ${ }^{1}$ MIIT Key Laboratory of Advanced Display Materials and Devices, Institute of Optoelectronics \& Nanomaterials, College of Materials \\ Science and Engineering, Nanjing University of Science and Technology, Nanjing 210094, China \\ ${ }^{2}$ Department of Materials Science and Engineering, University of Arizona, Tucson, Arizona 85721, USA
}

(Received 17 July 2019; published 29 January 2020)

\begin{abstract}
Achieving unidirectional emission and manipulating waves in a microcavity are crucial for information processing and data transmission in next-generation photonic circuits (PCs). Here we show how to impose twin microcavities with opposite chirality by incorporating parity-time $(\mathcal{P} \mathcal{T})$ symmetry to realize unidirectional emission. Our numerical calculation results show that the opposite chirality in microcavities stems from the asymmetric coupling of the clockwise $(\mathrm{CW})$ and counterclockwise $(\mathrm{CCW})$ components carried by the attached waveguide to the left- or right-sided microcavities, respectively. Notably, by engineering $\mathcal{P} \mathcal{T}$ symmetry in the coupled system via the gain-loss control, the clockwise component of the lossy cavity could be selectively suppressed, which leads to the unidirectional emission with an extinction ratio of up to $-52 \mathrm{~dB}$. Furthermore, the chirality and $\mathcal{P} \mathcal{T}$-symmetry breaking enabled unidirectional emission is extremely sensitive to external scatters, allowing the detection of nanoparticles with an ultrasmall radius of 5-50 $\mathrm{nm}$ by recording the extinction ratio change. The proposed system provides a simple yet general way to manipulate the standing waves in a microcavity and will be essential for advancing the potentials of the microcavity in PCs.
\end{abstract}

DOI: 10.1103/PhysRevA.101.013833

\section{INTRODUCTION}

The microcavity is one of the most attractive components for constructing PCs for various applications including highly sensitive sensors [1-3], isolators [4,5], and lasers [6-8]. Generally, the confined energy within the cavity will refractively leak from the cavity periphery; thus the coupling between the waveguide and the microcavity via evanescent waves is well suited to transport the signals into and out of the microcavity. Basically, the mirror reflection geometry symmetry of the microcavity sustains the coexistence of clockwise $(\mathrm{CW})$ and counterclockwise (CCW) standing waves with equal weights. Together with the rotational symmetry of the microcavity geometry, the standing-wave nature of the modes makes the emission from the microcavity omnidirectional in the far-field area. Many efforts have been devoted to overcome this limitation and achieve the effective manipulation of traveling waves in passive or active microcavity systems [9-11]. Although this feature causes no serious influence for most applications, the studies on chiral symmetry breaking in optical microcavity, which have been intensively explored theoretically and experimentally, are of great interest in both cavity physics and a range of applications from unidirectional lasers [12,13] and all-optical flip-flops [14] to highly sensitive sensors [15,16].

For this purpose, the rational way is to drive the microcavity to the status of chirality where the balanced weights of the $\mathrm{CW}$ or CCW component are broken. Thus far, several cate-

\footnotetext{
*zhiyuan.gu@njust.edu.cn

†ywang@njust.edu.cn
}

gories of techniques to address the issue have been intensively studied and well developed [17-20]. For instance, Liu et al. have demonstrated a unique and robust mechanism, which is assisted by chaotic-to-regular tunneling in dye-doped polymer microdisks with low refractive index, to realize the strong chirality in a deformed optical microcavity [17]. They found that the stable islands of the ray dynamics in the quadrupolar cavity are divided by the critical line $1 / n$ ( $n$ is the refractive index) in phase space. Hence the chaotic-to-regular tunneling dominates the escape path of the light, sequentially leading to asymmetric scattering and propelling the cavity to chirality. Different from stirring the chaos in a deformed cavity, Zhu and co-authors have developed a distinctive method to achieve chirality in a microcavity in the vicinity of the well-known exceptional points with multiple scatters on the rim [18], where multiple eigenvalues and eigenmodes coalesce and sensitivity is enhanced. Moreover, the deformed microdisks with chiral symmetry breaking have also been widely studied and verified by analyzing the rich physics in ray dynamics and wave function $[19,20]$. The created chiral boundary shape, such as the spiral microcavity [19] and the asymmetric Limaçon microcavity [20], can tune the cavity to chiral status that results in the outcoupling of whispering-gallery modes (WGMs) toward a particular direction.

In this work, we present a simple yet general approach to break the chiral symmetry in the microcavity-waveguide system, based on asymmetric scattering from waveguide to microcavities [21,22]. The traveling waves with counterrotation directions of the cavities are evanescently coupled to the waveguide; then the propagating light from one cavity in turn serves as an incoming wave to recouple into another 


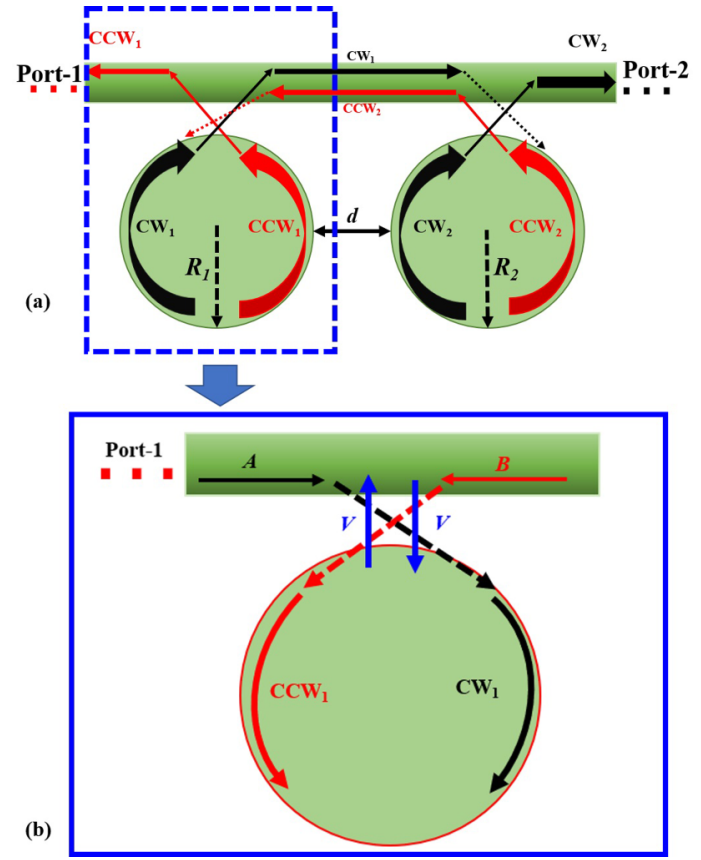

FIG. 1. (a) Schematics of the proposed coupling structure. The interval of two cavities and the gap between waveguide and microcavities are defined as $d$ and $h$, respectively. (b) The coupling between the waveguide and the left-sided cavity. The quantities $A$ and $B$ represent the incoming waves from the left and right sides of the waveguide, respectively.

microcavity. Under this asymmetric coupling from waveguide to the $\mathrm{CW}$ and $\mathrm{CCW}$ waves of the microcavities, strong chirality is constructed in the system. As a result, the emissions from the two ports of the waveguide are separately determined by $\mathrm{CW}$ and $\mathrm{CCW}$ components of the respective microcavity, allowing the manipulation of the traveling waves in the twin cavities. Interestingly, leveraging on the recently developed notion of $\mathcal{P} \mathcal{T}$ symmetry [23-27], the $\mathrm{CW}$ waves within the lossy cavity can be switched off, thus leaving alone the CCW traveling wave in the amplified cavity and giving unambiguous unidirectional emission with an extinction ratio as high as $-52 \mathrm{~dB}$. Notably, the designed structure shows superior sensitivity to the backscattering induced by nanoscale scatters, rendering the application in nanoparticle detection. By attaching a nanoparticle on the boundary of the amplified cavity, the 30-dB extinction ratio difference can be obtained when the size of the nanoparticle increases from 5 to $50 \mathrm{~nm}$.

\section{RESULTS AND DISCUSSIONS}

\section{A. Coupling in passive system}

The proposed structure is treated as a two-dimensional object with refractive index $n=n^{\prime}+i n^{\prime \prime}$, where the imaginary part $n^{\prime \prime}$ represents the gain $\left(n^{\prime \prime}>0\right)$ or loss $\left(n^{\prime \prime}<0\right)$. As shown in Fig. 1, a bus waveguide is coupled with the twin cavity and the edge-to-edge distance between them is defined as $h$ and the interval of twin cavities is $d$ [see Fig. 1(a)]. Here, the radius of the two cavities is set as $R_{1}=R_{2}=R$. The waveguide is assumed to be reflectiveless which can be experimentally achieved by fabricating grating couplers [28].
When the coupled system is on resonance, the $\mathrm{CW}$ and $\mathrm{CCW}$ waves with opposite rotation directions are simultaneously excited in each cavity, then independently couple into the waveguide, as illustrated in Fig. 1. Here, we define the two components of the left-sided (right-sided) cavity as $\mathrm{CW}_{1}$ $\left(\mathrm{CW}_{2}\right)$ and $\mathrm{CCW}_{1}\left(\mathrm{CCW}_{2}\right)$. For the left-sided microcavity, the $\mathrm{CW}_{1}$ component travels to the right direction, i.e., port 2 (the right port of the waveguide), whereas the $\mathrm{CCW}_{2}$ component of the right-sided cavity propagates toward port 1 (the left port of the waveguide). It is noted that the $\mathrm{CCW}_{1}$ and $\mathrm{CW}_{2}$ components leave the system as output energy; thus these two waves rarely affect the coupling process. Contrarily, the $\mathrm{CCW}_{2}$ modes from the right-sided cavity will be received by the left-sided cavity as incoming waves via the waveguide coupling and then interact with the $\mathrm{CCW}_{1}$ waves, as depicted in Fig. 1(b). Similarly, the $\mathrm{CW}_{1}$ waves from the left-sided cavity will be recoupled into the right-sided cavity and interact with the $\mathrm{CW}_{2}$ waves. For the left-sided cavity, however, the incoming light from its left side is almost zero due to the neglectable reflection from the waveguide. In this sense, it only receives the energy coming from the right side (i.e., the $\mathrm{CCW}_{2}$ component) of the waveguide, thus enhancing the weight of its $\mathrm{CCW}_{1}$ modes and breaking the chiral symmetry. Now we know that the quantities of the light flowing into the $\mathrm{CW}_{1}$ and $\mathrm{CCW}_{1}$ modes of the left-sided cavity are not equal, clearly forming the asymmetric scattering effect and generating strong chirality. The same asymmetric scattering process could also occur in the right-sided cavity but for the opposite rotation direction, i.e., the $\mathrm{CW}_{2}$ mode, eventually producing the inverse chirality with respect to the left-sided cavity. Though the combined system shows globally bidirectional emissions similar to the conventional case [29-31], the emission mechanism is exclusively different from the usual designs.

From the above analysis, it is known that the generation of the chirality in the microcavity is mainly dependent on the asymmetric couplings from the waveguide to the $\mathrm{CW}$ and CCW modes of the cavity, respectively. Hence, it is convenient to analyze the individual coupling process between one cavity and the side-coupled waveguide. Here we choose the left-sided cavity as an example to explore the underlying physics of the chirality. Figure 1(b) illustrates the interaction process between the waveguide and microcavity. Therefore, by defining the quantities $\Omega_{\mathrm{L}}$ and $\Omega_{\mathrm{WG}}$ as the complexvalued dimensionless frequencies of the left cavity modes and waveguide modes, the formation of chirality in the left-sided cavity can be qualitatively understood with the following $4 \times$ 4 non-Hermitian Hamiltonian [17,32,33].

$$
H=\left|\begin{array}{cccc}
\Omega_{\mathrm{L}} & V & 0 & 0 \\
V & \Omega_{\mathrm{WG}} & A & 0 \\
0 & B & \Omega_{\mathrm{WG}} & V \\
0 & 0 & V & \Omega_{\mathrm{L}}
\end{array}\right| .
$$

Here, the real-valued quantity $V$ is the coupling element describing the coupling between the left-sided cavity mode and the waveguide mode. The waveguide-to-cavity coupling occurs with exactly the same strength with the cavity-towaveguide coupling, because both processes are related by time-reversal symmetry. The complex-valued quantities $A$ and $B$ relate to the light within the waveguide flowing from left 
to right and its reverse process with respect to the left-sided cavity, respectively [see Fig. 1(b)]. Then, the eigenvalues of the above Hamiltonian are

$$
\begin{aligned}
\Omega_{ \pm, \rho}= & \frac{\Omega_{L}+\Omega_{\mathrm{WG}}+\rho \sqrt{A B}}{2} \\
& \pm \sqrt{V^{2}+\left(\frac{\Omega_{\mathrm{L}}-\Omega_{\mathrm{WG}}-\rho \sqrt{A B}}{2}\right)^{2}},
\end{aligned}
$$

with $\rho= \pm 1$. The non-normalized eigenvectors are

$$
\psi_{ \pm, \rho}=\left(\begin{array}{c}
\sqrt{A} \\
V_{ \pm, \rho} \sqrt{A} \\
\rho V_{ \pm, \rho} \sqrt{B} \\
\rho \sqrt{B}
\end{array}\right) .
$$

Here, the term $V_{ \pm, \rho}$ represents the abbreviation of $V_{ \pm, \rho}=$ $\frac{\Omega_{ \pm, \rho}-\Omega_{\mathrm{L}}}{V}$. In the spirit of the definition of the chirality $[32,33]$, we define the chirality of an eigenvector $\psi=\left(\psi_{1}, \psi_{2}, \psi_{3}, \psi_{4}\right)$ as

$$
\alpha_{1}=1-\frac{\min \left\{\left|\psi_{1}\right|^{2}+\left|\psi_{2}\right|^{2}, \quad\left|\psi_{3}\right|^{2}+\left|\psi_{4}\right|^{2}\right\}}{\max \left\{\left|\psi_{1}\right|^{2}+\left|\psi_{2}\right|^{2}, \quad\left|\psi_{3}\right|^{2}+\left|\psi_{4}\right|^{2}\right\}} .
$$

Plugging in the eigenvectors (2), we find

$$
\alpha_{1}=1-\frac{\min \{|A|,|B|\}}{\max \{|A|,|B|\}} .
$$

As mentioned in the beginning of this section, the energy propagating in the waveguide from right to left is larger than its reverse counterpart, that is, $A \neq B$; thus we get the asymmetric coupling from the waveguide to the $\mathrm{CW}_{1}$ and $\mathrm{CCW}_{1}$ components which consequently generates strong chirality in the left-sided cavity. Similarly, the above theoretical model can be applied to the right-sided microcavity and thus the corresponding chirality contrary to the left-sided cavity could be produced.

To check the above analysis, we numerically studied the interactions between the twin cavity and the waveguide based on the commercial finite-element-methods software (COMSOL MULTIPHYSICS 4.2A). In our simulation model, the real part of $n$ is set as $n=3.3$ (for InGaAsP) for transverse magnetic (TM; $E$ is perpendicular to the cavity plane) polarization. The perfectly matched layer (PML) is applied to fully absorb the outgoing waves. Then the far-field patterns (FFPs) of each mode are recorded by simulating the electromagnetic flux along the inner circle of the PML, which is $20 \lambda$ away from the center of the combined structure. The eigenfrequency module was employed to solve the resonant frequencies and field distributions of the modes. The calculated eigenfrequencies are defined as $f=f_{\text {real }}+i f_{\text {imag }}$, where $f_{\text {real }}$ and $f_{\text {imag }}$ represent the resonant frequency and linewidth, respectively. In order to clearly capture the physical insights behind the chirality, the passive situation is presented first. Here, the gap $h$ is set as $200 \mathrm{~nm}$ and the radius $R=2 \mu \mathrm{m}$ of the microcavity for demonstration purposes. To exclude the coupling between two cavities, the interval $d$ is chosen to be large enough, for instance, $2 \mu \mathrm{m}$. Figure 2(a) displays the resonant mode at $\lambda=$ $1014.6 \mathrm{~nm}$. Impressively, the two microcavities exhibit strong chirality and no clear nodal line is visible along the azimuthal direction, expressly confirming the chirality symmetry has been broken in these two resonators. Regarding the FFPs, the emission intensities of port 1 and port 2 are symmetrically distributed [see Fig. 2(b)] owing to the identical topological parameters of the two microcavities. In order to further uncover the essence of emissions from two ports and analyze the chirality, it is convenient to consider the angular momentum representation [34]. To do so, we expand the wave function inside the cavity in cylindrical harmonics,

$$
\psi(\rho, \phi)=\sum_{m=-\infty}^{\infty} \alpha_{m} J_{m}(n k \rho) \exp (\operatorname{im} \rho),
$$

where $J_{m}$ is the $m$ th-order Bessel function of the first kind, $k=\omega / c$ is the wave number and $\omega$ represents the eigenfrequency, and $(\rho, \phi)$ is the polar coordinate system as defined in Fig. 2(a). A positive (negative) value of the angular momentum index $m$ corresponds to the $\mathrm{CCW}(\mathrm{CW})$ propagating component.

The calculated angular distribution $\alpha_{m}$ of the mode in the left-sided cavity with Eq. (6) is shown in Fig. 2(c). We can observe that the angular momentum representation $\alpha_{m}$ is dominated by the $\mathrm{CCW}_{1}$ component and the mode can therefore be considered as a CCW traveling mode. On the contrary, the angular momentum representation $\alpha_{m}$ for the mode in the right-sided microcavity exhibits a sign change [see Fig. 2(d)], clearly verifying that the mode is governed by the $\mathrm{CW}$ traveling waves. To quantitatively characterize the chirality, we define the chirality as

$$
\alpha_{2}=1-\frac{\sum_{\mathrm{CW}} \text { Husimi }}{\sum_{\mathrm{CCW}} \text { Husimi }} .
$$

The Husimi function in Eq. (7) can be considered as the representation of the wave function on the Poincare surface of the section at the boundary of the dielectric cavities and has been widely used for the internal waves of the chaotic cavity $[35,36]$. In the passive case, the obtained chiralities for both the left and right cavities are $\alpha_{2} \approx 0.93$, explicitly expressing the fact that both modes have one strongly dominating propagation direction. Now, we can conclude that once the combined system is excited, the traveling waves in each cavity will rotate forever with opposite directions. To further understand the essential role of the waveguide, we have investigated the influence of the distance $h$ on the generated chirality of the system. The results are concluded in Fig. 2(e), where it is clearly seen that the chirality decreased monotonically with the increase of $h$. When $h$ is small, the coupling between the waveguide and two cavities is strong; thus the asymmetric coupling strengths from the waveguide to different components ( $\mathrm{CW}$ and $\mathrm{CCW}$ ) are large; i.e., the quantities $A$ and $B(A \neq B)$ are large, leading to the strong chirality of the two cavities. However, when the distance $h$ becomes large, though the relationship $A \neq B$ can still be sustained, the total energy coupled from waveguide into the cavities is reduced ( $A$ and $B \rightarrow 0$ ). Consequently, the asymmetric coupling induced chirality becomes weak. As we discussed before, the chirality stems from the asymmetric coupling from waveguide to cavities; thus the intervals between the two cavities are supposed to barely affect the generation of chirality. As demonstrated in Fig. 2(f), we calculated the 
(a)

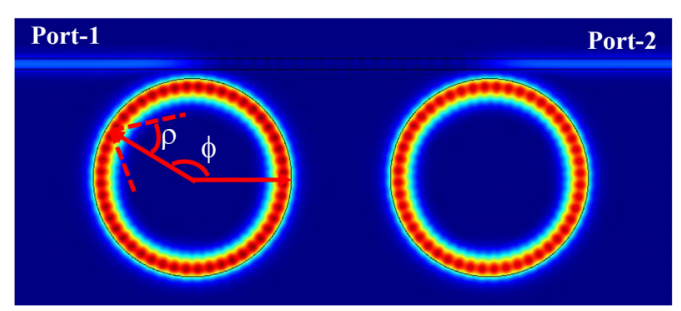

(c)
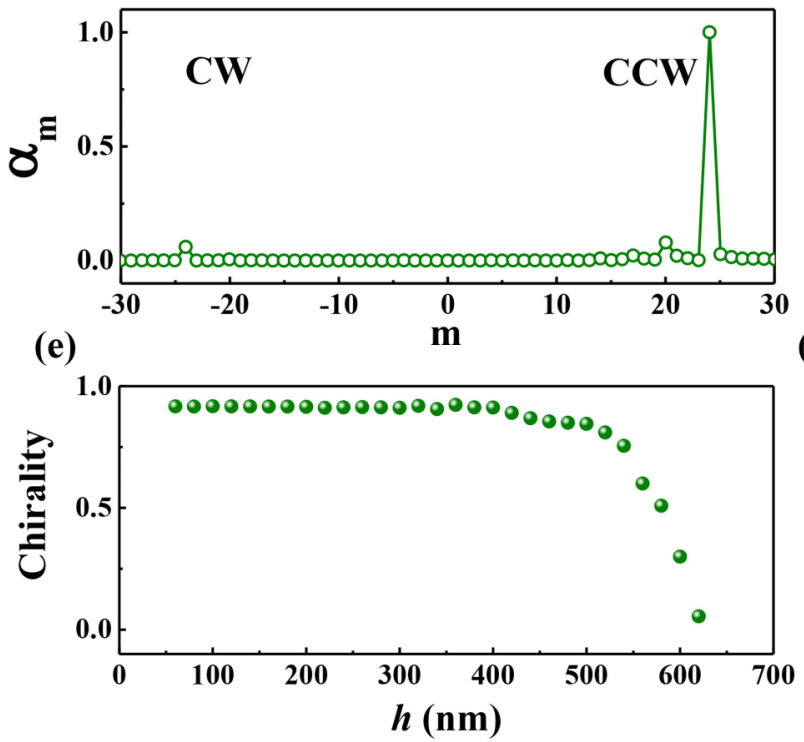

(b)

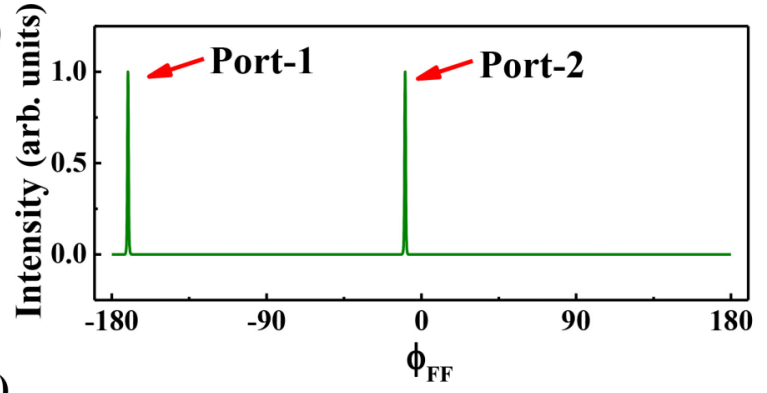

(d)
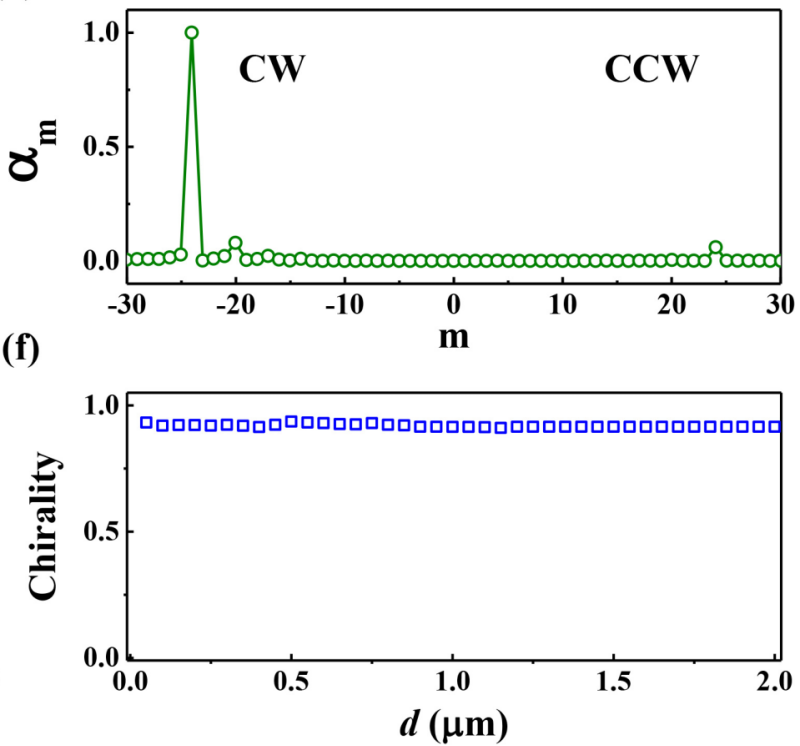

FIG. 2. Numerical results for passive system. (a) The electric-field distribution $|E|$ of resonant mode at $\lambda=1014.6 \mathrm{~nm}$. (b) The FFPs of the mode in (a). Angular momentum distributions $\alpha_{m}$ for the modes of the left- (c) and right-sided cavities (d), respectively. The chirality of the left-sided cavity as a function of the waveguide-microcavity distance $h$ (e) and the intervals $d$ (f) between the two cavities, respectively.

chirality of the left-sided cavity as a function of $d$. It is seen that the values of chirality almost maintain constant around $\alpha_{2} \approx 0.93$ as we expected.

Here, it is noted that the cavity radius should be much larger than the optical wavelength $\lambda$. Under this condition, the optical properties are determined by the intracavity ray dynamics [37-39]. Because for the case of wavelength-scale cavity, the classical ray model breaks down, and wave optical phenomena become significant. The degree of chirality will be impacted by the wave optical effects such as the "GoosHänchen" shift [12,40-42]. Besides, breaking the rotational symmetry of the cavity can also establish strong chirality $[34,43]$. However, the generated chirality is quite sensitive to the deformation parameters, making it difficult to demonstrate precisely in real experiments. Due to the chaotic characteristics of the deformed cavity, the mode identification is another issue with which we should be concerned. Therefore, the present work shows significant advantages in the simple mode analysis and facile experimental fabrication. Furthermore, the proposed mechanism to establish chirality is not limited by cavity shape and mode orders. As shown in Appendix A (Fig. 7), the strong chirality can also be obtained in the modes with radial order $l=2$ and $l=3$. Likewise, the deformed cavities with Limaçon and quadrupolar shapes exhibit the same broken chirality symmetry when evanescently coupled with a bus waveguide [see Fig. 8 in Appendix B].

\section{B. Coupling in $\mathcal{P} \mathcal{T}$-symmetric system}

For an isolated microcavity, it is hard to separately tailor the $\mathrm{CW}$ and $\mathrm{CCW}$ components due to the degenerate frequencies and spatial overlaps of them, thus inevitably leading to bidirectional emissions when coupled with waveguide [29-31]. Generally, the introduction of chiral designs such as spiral $[44,45]$ and annular cavity $[46,47]$ can facilely drive the resonators into chiral status. However, it should be noted that the undesired multiple modes in such structures will spoil the spectra purity and thus result in complex analysis of lasing behavior, especially in sensing applications [48]. Herein, by incorporating with $\mathcal{P} \mathcal{T}$ symmetry, we show that the unidirectional emission and single-mode lasing can be accomplished simultaneously in the proposed system. As aforementioned in Sec. II A, the gain and loss are achieved by setting the imaginary part of the complex refractive index to be positive or negative, which are $n=n^{\prime}+i n^{\prime \prime}$ and $n=n^{\prime}-$ $i^{\prime \prime}$, respectively, as shown in Fig. 3(a). In the computation models, $d$ and $h$ are fixed at $200 \mathrm{~nm}$; the radiuses of the cavities are $R_{1}=R_{2}=2 \mu \mathrm{m}$. The behaviors of the real parts of the resonant frequencies are concluded in Fig. 3(b). We can see that the frequency splitting of the lasing mode [red squares in Figs. 3(b) and 3(c)] and absorption mode [black spheres in Figs. 3(b) and 3(c)] monotonically decreases in the region where $0<n^{\prime \prime}<0.0012$, while degenerating at 
(a)

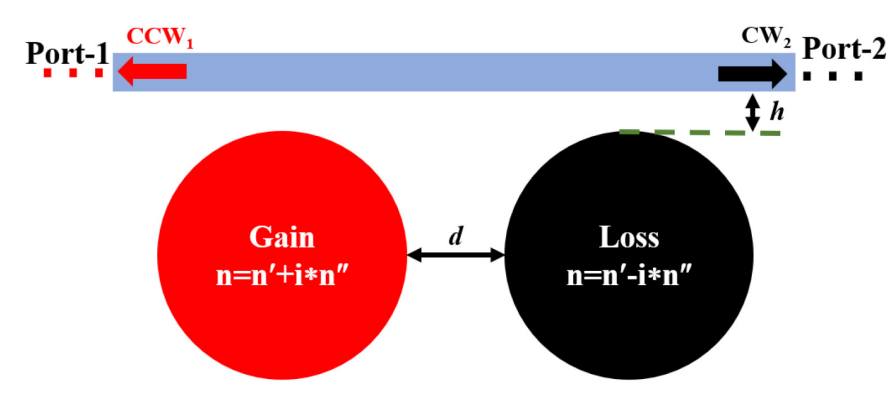

(c)

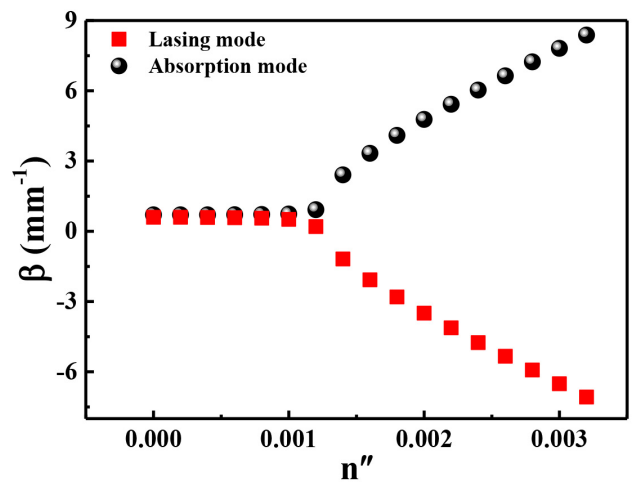

(b)

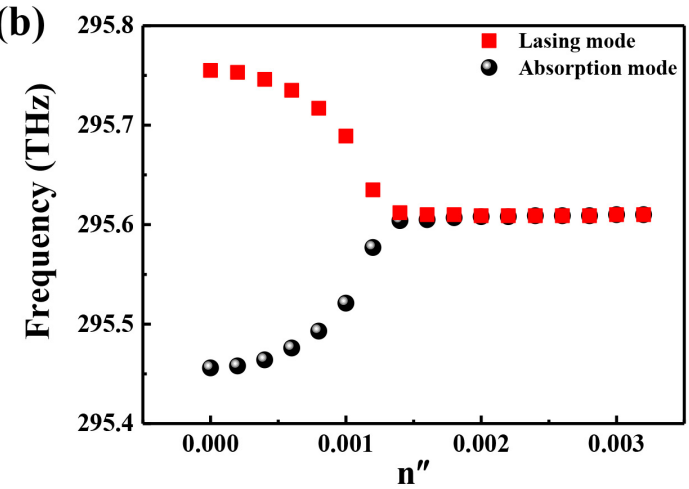

(d)

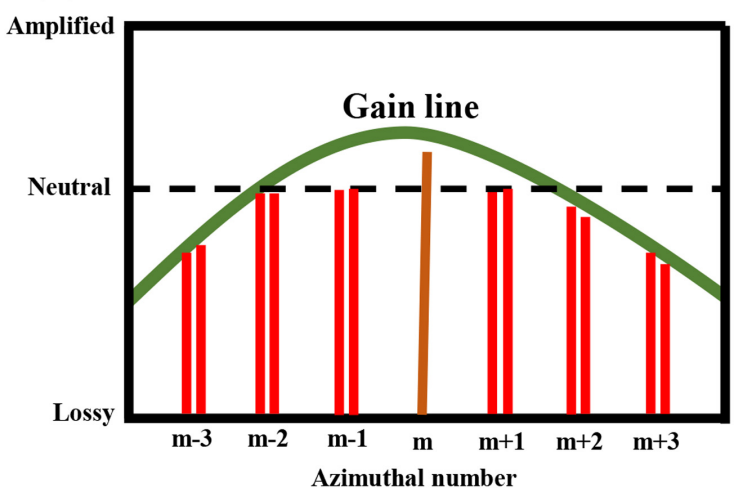

FIG. 3. (a) Schematics of the $\mathcal{P} \mathcal{T}$-symmetric microcavities. The evolutions of the real (b) and imaginary parts (c) of the eigenfrequencies as a function of gain factor $n^{\prime \prime}$. (d) The Lorentz distribution of the active medium. The center modes with azimuthal number $m$ obtain the largest gain and enter $\mathcal{P} \mathcal{T}$-symmetry breaking phase first.

$n^{\prime \prime}=0.0012$, and then is kept to be $295.6 \mathrm{THz}$ in the region with $0.0012<n^{\prime \prime}<0.003$. In contrast, the imaginary part $\beta$ ( $\beta=2 \pi f_{\text {imag }} / c ; c$ is the speed of light in vacuum) shows the opposite behavior to the real part [see Fig. 3(c)]. The $\beta$ of the two involved modes exhibits the same values in the region where $0<n^{\prime \prime}<0.0012$ and $n^{\prime \prime}=0.0012$. If the gain contrast is larger than 0.0012 , the degeneracy is broken in $\beta$. Furthermore, considering the Lorentz distribution of the gain spectra for the active material $[49,50$ ] [see olive curve in Fig. 3(d)], the resonant mode with azimuthal number $m$ located at the center of the gain window is supposed to owe the largest gain. As a result, the centered mode enters the $\mathcal{P} \mathcal{T}$-symmetry breaking threshold earlier and lases first. At the same time, the other modes are still experiencing the loss and existing in the unbroken $\mathcal{P} \mathcal{T}$-symmetry phase. In this sense, the gain that should have been fed to these modes will be plundered by the centered mode with the smallest $\mathcal{P} \mathcal{T}$-symmetry breaking threshold, consequently suppressing the lasing of other modes due to mode competition [51,52] and giving the single-mode lasing with enhanced gain [23,24].

In addition to the unconventional frequency features of the $\mathcal{P} \mathcal{T}$-symmetric microcavities, the dramatic changes in emissions from waveguide ends are more fascinating. In order to directly observe the changes in FFPs, the $\mathcal{P} \mathcal{T}$-symmetric case with $n^{\prime \prime}=0$ is presented first. As shown in Fig. 4(a), the mode energies are identically distributed in the cavity pair and the $\mathrm{CCW}_{1}$ and $\mathrm{CW}_{2}$ components together determine the emissions from the waveguide ends as mentioned above, reasonably leading to symmetric outputs from port 1 and port 2 [see the FFPs in Fig. 4(b)]. It is noted that the absorption mode with $\beta>0$ is undetectable experimentally; here we focus on the FFPs of lasing mode for simplicity. Given a larger gain strength with $n^{\prime \prime}=0.003$, the distinction between lasing mode and absorption mode is unambiguous. As revealed in Fig. 4(c), the mode photons of the lasing mode with $\mathrm{CCW}_{1}$ rotation are almost confined in the left cavity with gain, which dominates the emission propagating into port 1 . Because the emission from port 2 stems from the $\mathrm{CW}_{2}$ component of resonance in a lossy cavity where the light is significantly attenuated, thus the detected energy from port 2 is negligible. As confirmed by the FFPs in Fig. 4(d), the intensity ratio of port 2 and port 1 is as small as 0.04 (corresponding to an extinction ratio of $-33 \mathrm{~dB}$ ), which can be further improved by optimizing the structure parameters. We thus can conclude that the $\mathrm{CW}_{2}$ waves could be effectively eliminated leveraging on sufficient gain/loss contrast in the two-microcavity-waveguide system, impressively inducing asymmetric outputs.

\section{Optimization of extinction ratio in $\mathcal{P} \mathcal{T}$-symmetric system}

Principally, the asymmetric coupling $(A \neq B)$ accounts for the strong chirality constructed in the twin microcavity. Considering that the coupling strength relates light exchange between waveguide and microcavities is a critical issue which directly affects the degree of chirality. In this sense, it is vital to investigate the dependence of coupling on the intervals between these elements. Basically, the eventual unidirectional emission in the $\mathcal{P} \mathcal{T}$-symmetric microcavity is simultaneously 
(a)

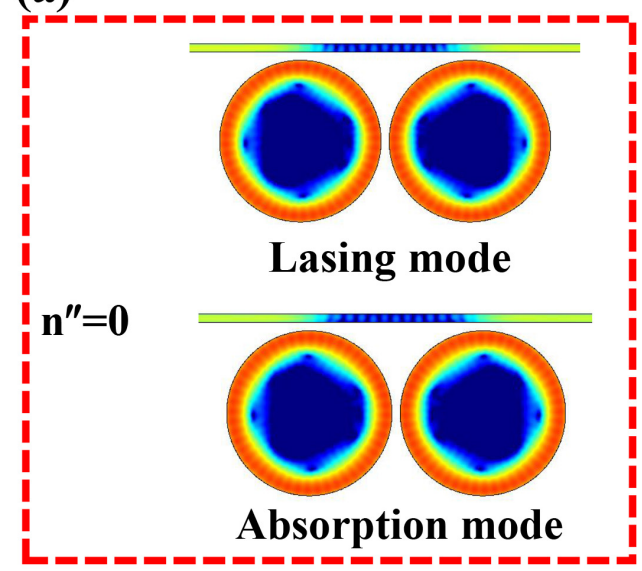

(c)

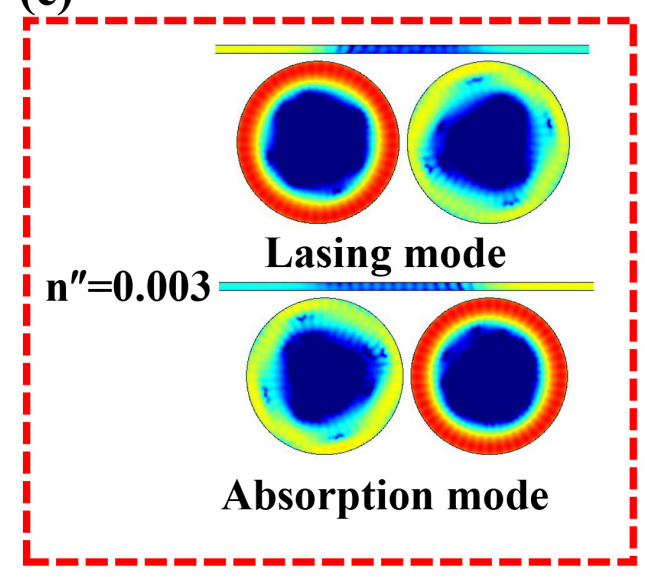

(b)

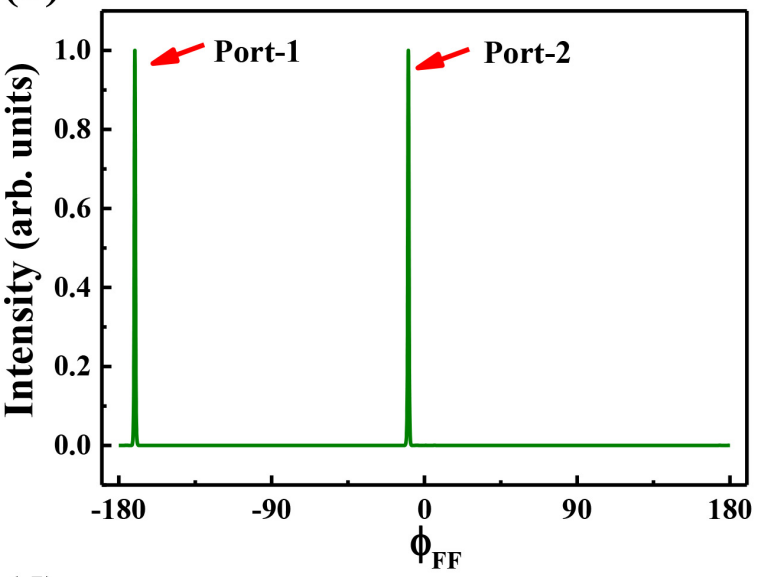

(d)

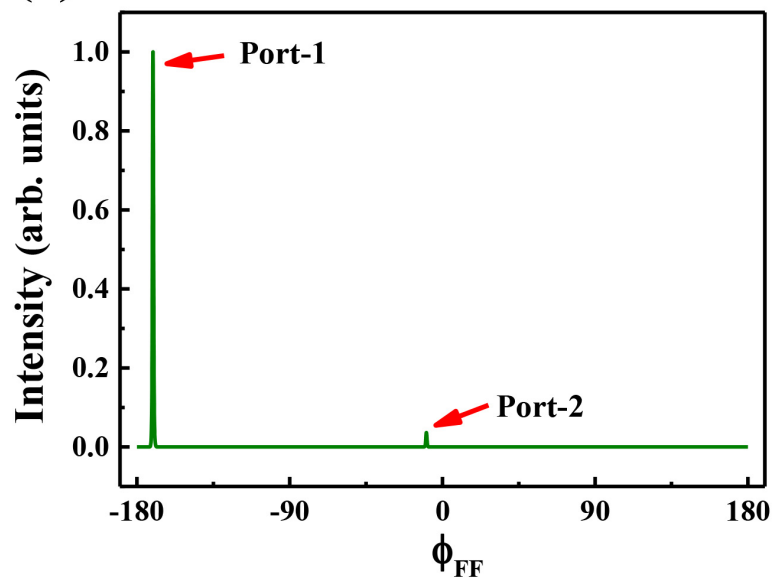

FIG. 4. The mode field distributions of the lasing mode and absorption for $n^{\prime \prime}=0$ (a) and 0.003 (c). The FFPs of the lasing modes with $n^{\prime \prime}=0$ (b) and 0.003 (d). Here the interval of two cavities $d$ and the distance between waveguide and microcavities $h$ are fixed at 300 and $200 \mathrm{~nm}$, respectively.

determined by two parts of coupling. The first one is the evanescent coupling between waveguide and cavities, which is related to $h$. To crisply uncover the influence of the distance $h$ on unidirectional emission, we fixed the interval of two cavities $d$ as $300 \mathrm{~nm}$ while varying $h$. The recorded extinction ratios of port 1 and port 2 are summarized in Fig. 5(a); we can see that the extinction ratio steadily decreases with the increase of $h$. This is because the coupling efficiency between waveguide and cavities becomes weaker when $h$ gets larger; thus the total energy participating in the process of asymmetric coupling is limited and the chirality sequentially becomes smaller. As a result, the uncoupled $\mathrm{CW}_{1}$ component within the waveguide will be directly delivered to port 2; thus the extinction ratio of port 2 and port 1 becomes large. If the distance $h$ is small enough, for instance, $h=60 \mathrm{~nm}$, an extinction ratio as high as $-52 \mathrm{~dB}$ can be achieved. Notably, the extinction ratio can be maintained less than $-20 \mathrm{~dB}$ in the range where $50 \mathrm{~nm}<h<380 \mathrm{~nm}$, hinting toward the low requirement of fabrication resolution and acceptable operation errors in the experimental demonstrations.

Another part of coupling is the interactions between two microcavities. As shown in Fig. 5(b), the extinction ratios experience decreases in the range where $100 \mathrm{~nm}<d<340 \mathrm{~nm}$ with fixed $h=200 \mathrm{~nm}$. However, when $d$ exceeds $340 \mathrm{~nm}$, the extinction ratios climb slowly with the increase of $h$. As we discussed above, the unidirectional emission with an distinctive extinction ratio of the proposed system originates from the applied gain and loss contrast, i.e., the $\mathcal{P} \mathcal{T}$-symmetry breaking induced suppression of the mode within a lossy cavity. As reported in previous works [24,53,54], the $\mathcal{P} \mathcal{T}$ symmetry breaking threshold is associated with the relationship of coupling coefficient $\kappa$ and the gain $\gamma$. Therefore, it is essential to investigate the influence of the parameter $\kappa$ (by varying the distance $d$ ) on the $\mathcal{P} \mathcal{T}$-symmetry threshold where $\kappa=\gamma$. The results are concluded in Fig. 5(c) (blue triangles). We can see that the breaking threshold decreases with the increase of the distance $d$, i.e., the decrease of coupling strength $\kappa$, which is consistent with the previous reports $[24,53,54]$. When the system is applied with a gain strength $\gamma$, the configurations with larger $d$ will enter the $\mathcal{P} \mathcal{T}$-symmetry breaking phase first. In this sense, the suppression of the lossy mode should be more dramatic compared to the situations with smaller $d$. Thus, the extinction ratio first exhibits declining with the increase of $d$. When the distance $d$ gets even larger, the $\mathcal{P} \mathcal{T}$-symmetry breaking threshold becomes smaller, resulting in the drastic suppression of the lossy mode. However, such a highly suppressed mode in the right-sided cavity will certainly reduce the quantity $B$, thus consequently 

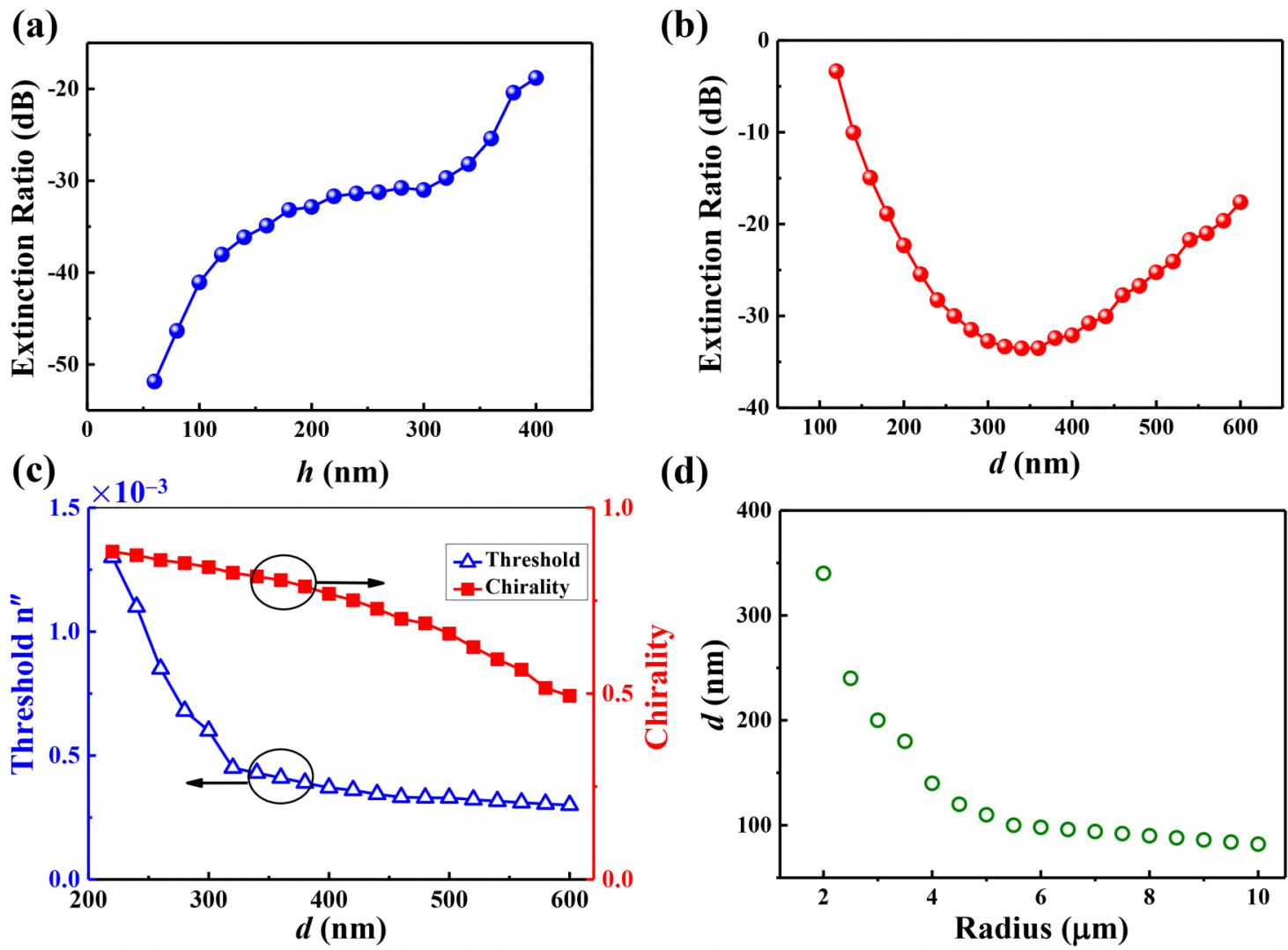

FIG. 5. (a) The dependence of extinction ratios as a function of distance between waveguide and microcavities $h$. Here the interval $d$ of two cavities is fixed at $300 \mathrm{~nm}$. (b) The dependence of extinction ratios on the interval $d$, while fixing $h$ as $200 \mathrm{~nm}$. (c) Blue triangles: evolution of the $\mathcal{P} \mathcal{T}$-symmetry breaking threshold when the coupling distance $d$ between two resonators is varied. Red squares: dependence of chirality of the left-sided cavity on the coupling distance $d$. (d) Optimized coupling distance $d$ for the waveguide-cavity system with different cavity radius.

deteriorating the chirality induced by asymmetric coupling from the waveguide. As shown in Fig. 5(c) (red squares), the chirality of the left-sided cavity decreases when the distance $d$ gets larger. According to the analysis in Sec. II A, we know that both the chirality and $\mathcal{P} \mathcal{T}$ symmetry determine the globally unidirectional emission of the system. Therefore, though the suppression of the lossy mode is stronger, the chirality is reduced, accordingly making the extinction ratio increase when $d>340 \mathrm{~nm}$. Then the behaviors of the extinction ratios at different $d$ are understandable. It is the trade-off between $\mathcal{P} \mathcal{T}$ symmetry and chirality of the combined system when the distance $d$ changes. For the parameter $d$ with the region from 200 to $550 \mathrm{~nm}$, we can see that the extinction ratio is lower than $-20 \mathrm{~dB}$, thus allowing a wide range of fabrication deviation while maintaining the high performance of the device. To provide the design parameters for the experimental demonstration, we also optimize the microcavities intervals $d$ under different radii to achieve the best extinction ratio of the system. The results are summarized in Fig. 5(d); it is seen that the distance $d$ decreases with the increase of the cavity radius. For larger cavity radius, the total loss is smaller, then the $\mathcal{P} \mathcal{T}$-symmetry breaking threshold should be less than that of cavity with smaller radius; thus the suppression of the lossy mode would be stronger. In the above discussion, we know that the highly suppressed mode will reduce the chirality of the cavity. In this sense, to maintain the smallest extinction ratio of the system with larger cavity radius, the $\mathcal{P} \mathcal{T}$-symmetry threshold consequently needs to be improved which can be obtained by increasing the coupling strength $\kappa$, i.e., decreasing the distance $d$.

\section{Nanoparticle sensing in $\mathcal{P} \mathcal{T}$-symmetric system}

Since the chirality stems from the broken symmetry of $\mathrm{CW}$ and $\mathrm{CCW}$ waves, the introduction of backscattering induced by external perturbations is an archetypical approach to seize back the coupling between them, implying the potential application of chirality in sensing [55]. In this section, we explore the possibility of the proposed system in nanoparticle sensing by breaking the chirality. Figure 6(a) shows a schematic drawing of the system. A nanoparticle of radius $r$ is placed near the boundary of the gain cavity with a $20-\mathrm{nm}$ gap. Here the distance $h$ and interval $d$ are set as 100 and $300 \mathrm{~nm}$, respectively. The calculated chirality $\alpha_{2}$ for different radius $r$ is displayed in Fig. 6(b). When the radius $r$ is $5 \mathrm{~nm}$, the generated backscattering is tiny $\left(\alpha_{2} \approx 0.86\right)$, thus weakly reducing the chirality. As the mode field pattern shown in Fig. 6(c), the interference fringes of the lasing mode within a left-sided cavity are almost invisible, which exactly confirms the unbalanced distribution of $\mathrm{CW}$ and $\mathrm{CCW}$ components 
(a)

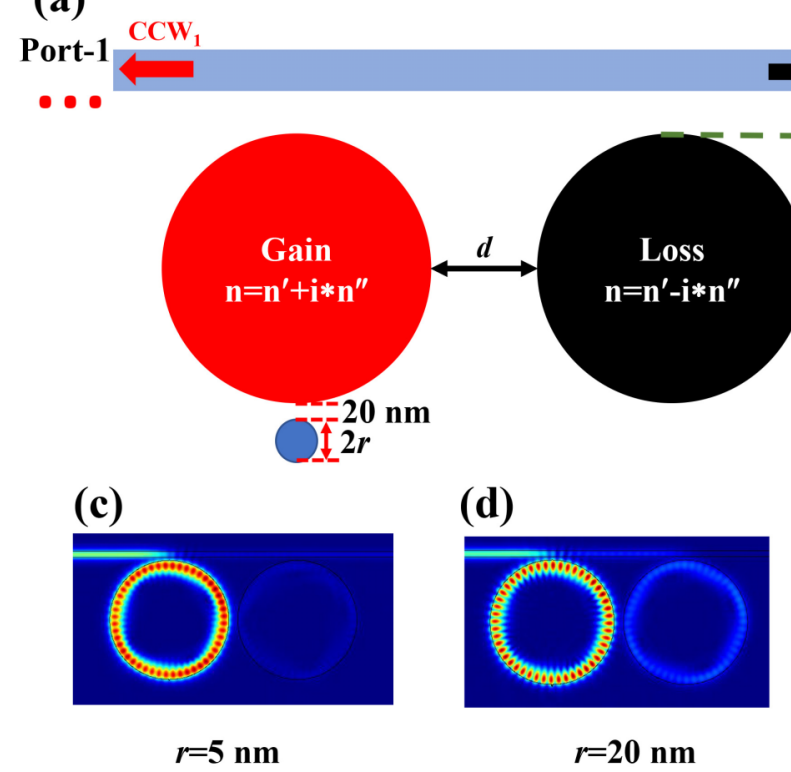

(b)

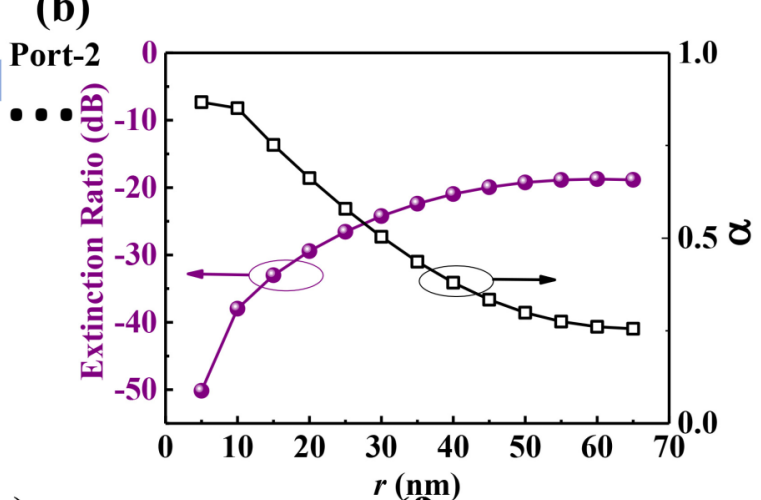

(e)

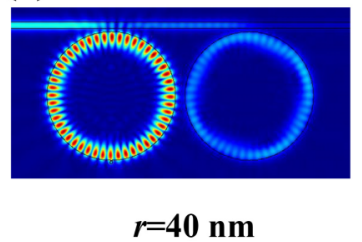

(f)

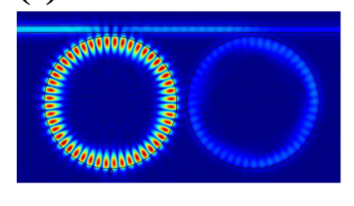

$r=65 \mathrm{~nm}$

FIG. 6. Nanoparticle detection in the microcavity. (a) The scheme of the detection system. Here the edge-to-edge distance between particle and microcavity is $20 \mathrm{~nm}$. (b) The extinction ratios and chirality as a function of nanoparticle size $r$. (c-f) The mode field distributions with $r=5 \mathrm{~nm}, 20 \mathrm{~nm}, 40 \mathrm{~nm}$, and $65 \mathrm{~nm}$, respectively.

and consequently leads to an extinction ratio of $-50 \mathrm{~dB}$. In this sense, the mode is not standing waves but mainly traveling waves. However, if the radius $r$ increases to 20 $\mathrm{nm}$, the backscattering stimulates the bidirectional coupling between $\mathrm{CW}_{1}$ and $\mathrm{CCW}_{1}$ waves and the nodes along the azimuthal direction are distinguishable $\left(\alpha_{2} \approx 0.662\right)$. Hence, the extinction ratio drastically increases from $-52 \mathrm{~dB}$ to $-29 \mathrm{~dB}$ owing to the contribution of $\mathrm{CW}_{1}$ waves at port 2 . When the radius $r$ further increases to $40 \mathrm{~nm}$, the mode field pattern [see Fig. 6(e)] is similar to the usual standing-wave modes reported by many other groups [18]. In this case, the chirality reduces to $\alpha_{2} \approx 0.38$ which achieves an extinction ratio of $-21 \mathrm{~dB}$. For the nanoparticle radius of $r=65 \mathrm{~nm}\left(\alpha_{2} \approx 0.255\right)$, the node patterns are clearer due to strong Rayleigh scattering [55] that sufficiently deteriorates the unidirectional emission and thus reduces the extinction ratio to $-18.8 \mathrm{~dB}$. From Fig. 6(b), we can see that the Rayleigh scattering strength is enhanced with the increase of particle size, accordingly leading to the increase of extinction ratios and decrease of chirality $\alpha_{2}$. It is worth noting that the decrease of the extinction ratio in the region where $5 \mathrm{~nm}<r<50 \mathrm{~nm}$ is rapid, while that in the region with $50 \mathrm{~nm}<r<65 \mathrm{~nm}$ slightly changes. This is because the perturbations-evoked backscattering is almost saturated for $50 \mathrm{~nm}<r<65 \mathrm{~nm}$ which slows down the escalating trend of the extinction ratio. Furthermore, compared with conventional microcavity sensors using mode splitting [56] or far-field patterns [29], the sensing mechanism of our structure shows unambiguous advantages. First, a highresolution $(\Delta v \sim 100 \mathrm{kHz})$ spectrometer is not required to analyze the spectra information. Second, a rotational translation stage is not necessary to resolve the change of the far-field emission intensities, thus efficiently reducing the operation error and experimental uncertainties. In the experimental realization, the in situ measurements of the proposed micro- cavity sensors in this work should be more convenient and robust.

\section{CONCLUSION}

In summary, we have proposed and investigated a twomicrocavity-waveguide system that shows strong chirality which is not limited by cavity shape and mode order. The symmetry breaking of chirality is attributed to asymmetric coupling which is clearly identified by the corresponding theoretical model and the numerical calculations. Moreover, with the assistance of $\mathcal{P} \mathcal{T}$ symmetry, the combined structure exhibits unidirectional emission with an extinction ratio lower than $-20 \mathrm{~dB}$ in the region with $50 \mathrm{~nm}<h<380 \mathrm{~nm}$ and $200 \mathrm{~nm}<d<550 \mathrm{~nm}$, obviously mitigating the requirement of fabrication precision to obtain chirality. According to the mechanism of unidirectional emission, we extend our system to the application of nanoparticle detection, a nano scatter of radius $5 \mathrm{~nm}<r<50 \mathrm{~nm}$ can be apparently felt by comparing the extinction ratio of the emission from port 1 and port 2 . We believe that the proposed microcavity-waveguide design may provide various potential applications in manipulating the traveling waves of the microcavity and play important role in on-chip PCs for optical interconnects and switching.

\section{ACKNOWLEDGMENTS}

This work was supported by the financial support from the Postdoctoral Science Foundation (Grant No. 2017M621274), the NSFC (Grants No. 61905113, and No. 11904172), the Natural Science Foundation of Jiangsu Province (Grant No. BK20190446), the One-Thousand Talent programs from the Chinese government, and the start-up funding from Nanjing University of Science and Technology. 

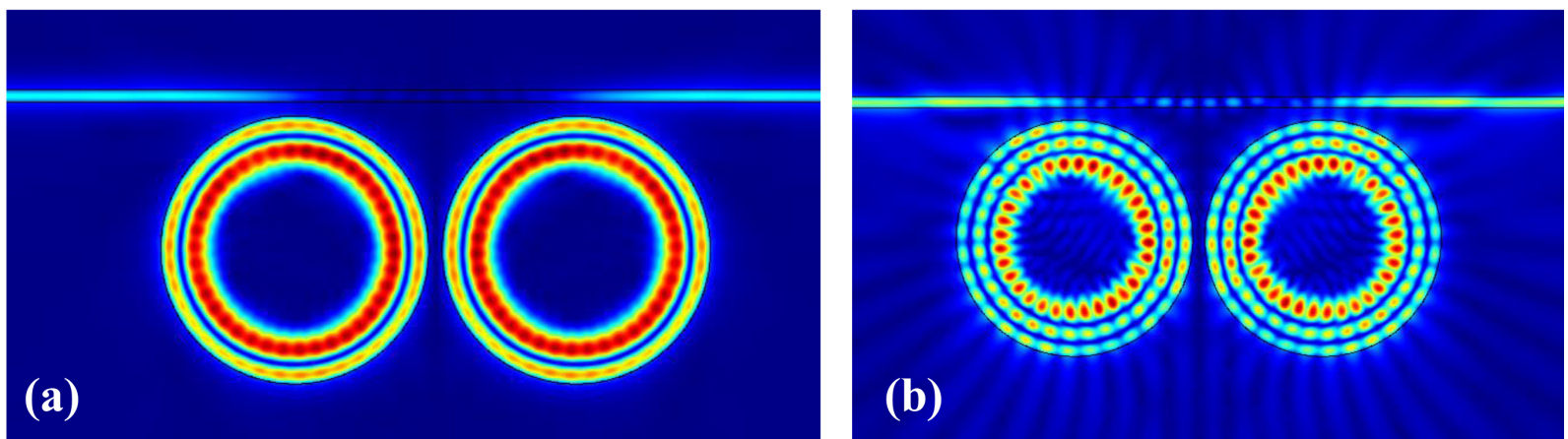

FIG. 7. The mode field patterns for the high-order modes with $l=2$ (a) and $l=3$ (b) of the circular microcavities.

\section{APPENDIX A: CHIRALITY OF HIGH-ORDER MODES IN} CIRCULAR MICROCAVITY

In this Appendix, we will examine the robustness of the asymmetric coupling induced chirality of high-order modes in a circular microcavity. All the structure parameters are the same as that in Fig. 2 in the main text. It is clearly seen that strong chirality has been established in the mode with $l=2$ and $l=3$. The nodal lines along the azimuthal direction for $l=2$ are almost invisible. It is seen that the energy density in the waveguide between two cavities is almost neglectable, clearly confirming the asymmetric coupling in this region. Likewise, the chirality holds true for the third-order modes with $l=3$ as shown in Fig. 7 .

\section{APPENDIX B: CHIRALITY IN DEFORMED MICROCAVITY}

Similarly, the strong chirality in a deformed microcavity can also be observed by constructing asymmetric coupling in the bus waveguide. As shown in Fig. 8(a), the shape of the quadrupolar cavity is described as $\rho(\theta)=R[1+\varepsilon \cos (2 \theta)]$.
Here the radius $R=5 \mu \mathrm{m}$ and the deformation factor $\varepsilon$ is fixed at 0.08 . We can see that the nodal line along the quadrupole orbit is hardly recognized and the energy density within the coupling area is quite weak, whereas the intensities around the waveguide emission ports are strong because the ray dynamics in the deformed cavity is complicated. We perform the control calculations for the system without the participation of the waveguide so as to verify the role of the waveguide in the system. As shown in Fig. 8(b), the well-defined nodes of standing waves in the cavity can be seen, demonstrating the critical function of the waveguide in constructing chirality. As we claimed in the main text, the proposed mechanism is not limited by the shape of the microdisks. We have also observed the same phenomenon in a Limaçon cavity, as revealed in Fig. 8(c). The shape of the Limaçon cavity is determined by $\rho(\theta)=R[1+\varepsilon \cos (\theta+\pi)]$. Here the deformation $\varepsilon=0.4$. Likewise, the absence of the waveguide also leads to the predominance of standing waves in the cavity as expected. As confirmed by Fig. 8(d), clear interference patterns show that the mode is dominated by the standing waves in both microcavities.
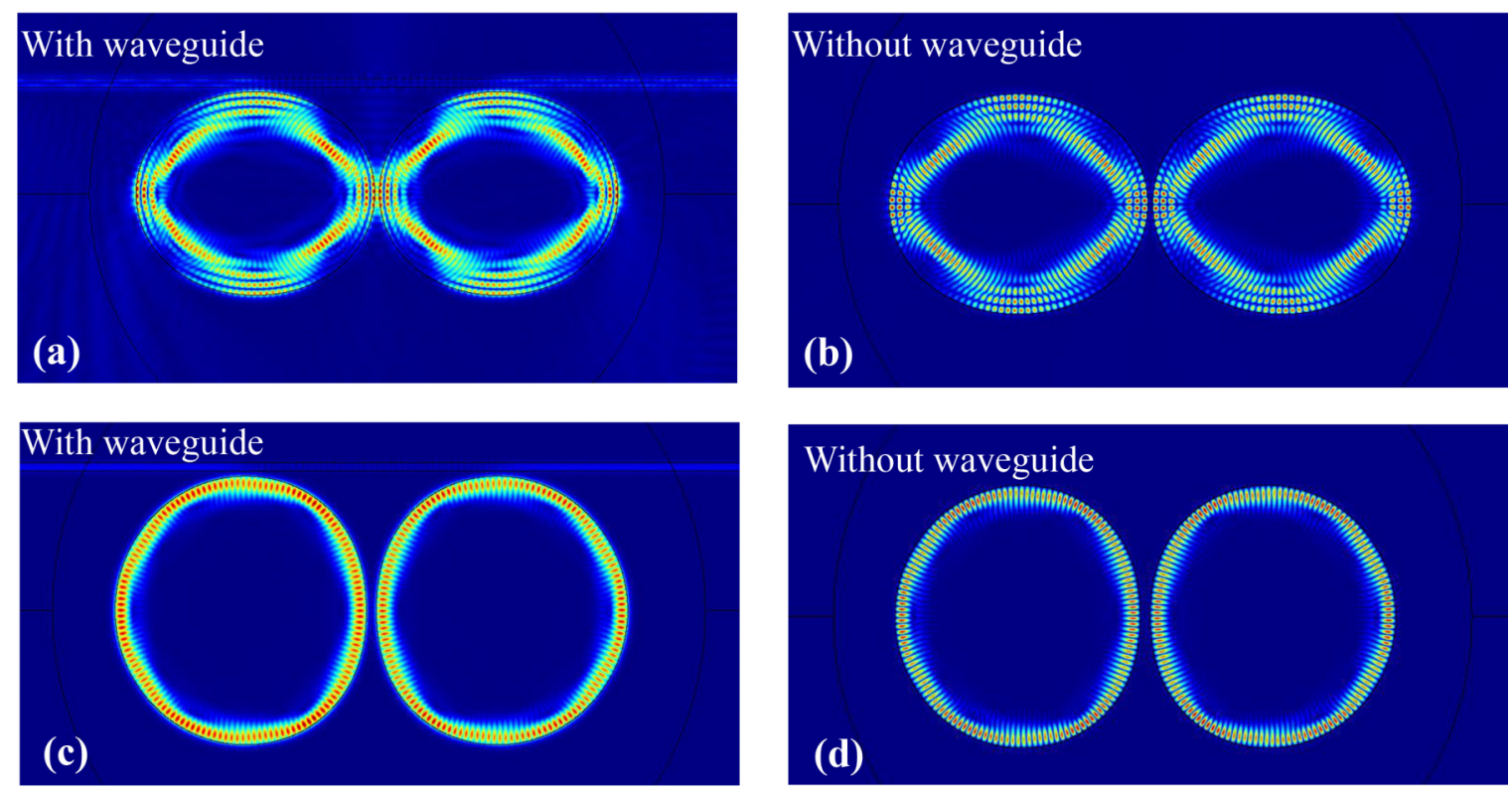

FIG. 8. The mode field distributions with (a) and without (b) waveguide coupling in the quadrupolar microcavity. The mode field distributions with (c) and without (d) waveguide coupling in the Limaçon microcavity. 
[1] W. J. Chen, Ş. K. Özdemir, G. M. Zhao, J. Wiersig, and L. Yang, Nature 548, 192 (2017).

[2] L. B. Shao, X.-F. Jiang, X.-C. Yu, B.-B. Li, W. R. Clements, F. Vollmer, W. Wang, Y.-F. Xiao, and Q. H. Gong, Adv. Mater. 25, 5616 (2013).

[3] Y. Y. Zhi, X.-C. Yu, H.-J. Chen, B.-O. Guan, and Y.-F. Xiao, Opt. Lett. 44, 2426 (2019).

[4] S. Y. Hua, J. M. Wen, X. S. Jiang, Q. Hua, L. Jiang, and M. Xiao, Nat. Commun. 7, 13657 (2016).

[5] N. Kono, K. Kakihara, K. Saitoh, and M. Koshiba, Opt. Express 15, 7737 (2007).

[6] C. Huang, W. Z. Sun, S. Liu, S. Li, S. Wang, Y. J. Wang, N. Zhang, H. B. Fu, S. M. Xiao, and Q. H. Song, Laser Photonics Rev. 13, 1800189 (2019).

[7] Y. Wang, Z. Y. Gu, Y. J. Ren, Z. M. Wang, B. Q. Yao, Z. L. Dong, G. Adamo, H. B. Zeng, and H. D. Sun, ACS Appl. Mater. Inter. 11, 15756 (2019).

[8] F. X. Gu, F. M. Xie, X. Lin, S. Y. Linghu, W. Fang, H. P. Zeng, L. M. Tong, and S. L. Zhuang, Light Sci. Appl. 6, e17061 (2017).

[9] B. Redding, L. Ge, G. S. Solomon, and H. Cao, Appl. Phys. Lett. 100, 061125 (2012).

[10] D. Liang, S. Srinivasan, D. A. Fattal, M. Fiorentino, Z. Huang, D. T. Spencer, J. E. Bowers, and R. G. Beausoleil, IEEE Photonics Technol. Lett. 24, 1988 (2012).

[11] X. Cai, G. Mezosi, M. Sorel, Z. Wang, Y. Shu, P. Jiang, and S. Yu, IEEE Photonics Technol. Lett. 23, 1636 (2011).

[12] B. Redding, L. Ge, Q. H. Song, J. Wiersig, G. S. Solomon, and H. Cao, Phys. Rev. Lett. 108, 253902 (2012).

[13] B. Peng, S. K. Özdemir, M. Liertzer, W. Chen, J. Kramer, H. Yilmaz, J. Wiersig, S. Rotter, and L. Yang, Proc. Natl. Acad. Sci. USA 113, 6845 (2016).

[14] M. T. Hill, H. J. S. Dorren, T. de Vries, X. J. M. Leijtens, J. H. den Besten, B. Smalbrugge, Y.-S. Oei, H. Binsma, G.-D. Khoe, and M. K. Smit, Nature 432, 206 (2004).

[15] M. D. Baaske, M. R. Foreman, and F. Vollmer, Nat. Nanotechnol. 9, 933 (2014).

[16] B. Q. Shen, X. C. Yu, Y. Zhi, L. Wang, D. Kim, Q. Gong, and Y.-F. Xiao, Phys. Rev. Appl. 5, 024011 (2016).

[17] S. Liu, J. Wiersig, W. Z. Sun, Y. B. Fan, L. Ge, J. Yang, S. M. Xiao, Q. H. Song, and H. Cao, Laser Photonics Rev. 12, 1800027 (2018).

[18] J. G. Zhu, Ş. K. Özdemir, L. He, and L. Yang, Opt. Express 18, 23535 (2010).

[19] J. Ryu, J.-W. Lee, C.-H. Yi, J.-H. Kim, I.-G. Lee, H.-S. Kim, S.-B. Kim, K. R. Oh, and C.-M. Kim, Opt. Express 25, 3381 (2017).

[20] N. C. Zambon, P. St-Jean, M. Milićević, A. Lemaître, A. Harouri, L. L. Gratiet, O. Bleu, D. D. Solnyshkov, G. Malpuech, I. Sagnes, S. Ravets, A. Amo, and J. Bloch, Nat. Photonics 13, 283 (2019).

[21] J. Wiersig, Phys. Rev. Lett. 97, 253901 (2006).

[22] Q. H. Song, L. Ge, J. Wiersig, and H. Cao, Phys. Rev. A 88, 023834 (2013).

[23] C. M. Bender and S. Boettcher, Phys. Rev. Lett. 80, 5243 (1998).

[24] H. Hodaei, M.-A. Miri, M. Heinrich, D. N. Christodoulides, and M. Khajavikhan, Science 346, 975 (2014).
[25] C. E. Rüter, K. G. Makris, R. El-Ganainy, D. N. Christodoulides, M. Segev, and D. Kip, Nat. Phys. 6, 192 (2010).

[26] L. Feng, Y.-L. Xu, W. S. Fegadolli, M.-H. Lu, J. E. B. Oliveira, V. R. Almeida, Y.-F. Chen, and A. Scherer, Nat. Mater. 12, 108 (2013).

[27] L. Ge and A. D. Stone, Phys. Rev. X 4, 031011 (2014).

[28] X. B. Luo, J. H. Huang, H. H. Zhong, X. Z. Qin, Q. T. Xie, Y. S. Kivshar, and C. H. Lee, Phys. Rev. Lett. 110, 243902 (2013).

[29] N. Zhang, Z. Y. Gu, S. Liu, Y. J. Wang, S. Wang, Z. H. Duan, W. Z. Sun, Y.-F. Xiao, S. M. Xiao, and Q. H. Song, Optica 4, 1151 (2017).

[30] P. J. Cegielski, A. L. Giesecke, S. Neutzner, C. Porschatis, M. Gandini, D. Schall, C. A. R. Perini, J. Bolten, S. Suckow, S. Kataria, B. Chmielak, T. Wahlbrink, A. Petrozza, and M. C. Lemme, Nano Lett. 18, 6915 (2018).

[31] H. T. Hattori, C. Seassal, E. Touraille, P. Rojo-Romeo, X. Letartre, G. Hollinger, P. Viktorovitch, L. Di Cioccio, M. Zussy, L. E. Melhaoui, and J. M. Fedeli, IEEE Photonics Technol. Lett. 18, 223 (2006).

[32] J. Wiersig, Phys. Rev. A 84, 063828 (2011).

[33] J. Wiersig, Phys. Rev. Lett. 112, 203901 (2014).

[34] J. Wiersig, A. Eberspächer, J.-B. Shim, J.-W. Ryu, S. Shinohara, M. Hentschel, and H. Schomerus, Phys. Rev. A 84, 023845 (2011).

[35] M. Hentschel, H. Schomerus, and R. Schubert, Europhys. Lett. 62, 636 (2003).

[36] H. E. Tureci, H. G. L. Schwefel, P. Jacquod, and A. D. Stone, Prog. Opt. 47, 75 (2005).

[37] X. F. Jiang, Y.-F. Xiao, C.-L. Zou, L. N. He, C.-H. Dong, B.-B. Li, F.-W. Sun, L. Yang, and Q. H. Gong, Adv. Mater. 24, OP260 (2012).

[38] H. G. L. Schwefel, N. B. Rex, H. E. Tureci, R. K. Chang, A. D. Stone, T. Ben-Messaoud, and J. Zyss, J. Opt. Soc. Am. B 21, 923 (2004).

[39] S. Shinohara, T. Harayama, T. Fukushima, M. Hentschel, T. Sasaki, and E. E. Narimanov, Phys. Rev. Lett. 104, 163902 (2010).

[40] Q. H. Song, L. Ge, A. D. Stone, H. Cao, J. Wiersig, J.-B. Shim, J. Unterhinninghofen, W. Fang, and G. S. Solomon, Phys. Rev. Lett. 105, 103902 (2010).

[41] N. B. Rex, H. E. Tureci, H. G. L. Schwefel, R. K. Chang, and A. D. Stone, Phys. Rev. Lett. 88, 094102 (2002).

[42] H. Schomerus and M. Hentschel, Phys. Rev. Lett. 96, 243903 (2006).

[43] J. Wiersig, S. W. Kim, and M. Hentschel, Phys. Rev. A 78, 053809 (2008).

[44] G. D. Chern, H. E. Tureci, A. D. Stone, and R. K. Chang, Appl. Phys. Lett. 83, 1710 (2003).

[45] X. S. Luo and A. W. Poon, Opt. Express 15, 17313 (2007).

[46] Q. H. Song, Z. Y. Gu, N. Zhang, K. Y. Wang, N. B. Yi, and S. M. Xiao, Sci. China Phys. Mech. 58, 114210 (2015).

[47] S.-Y. Lee, S. Rim, J.-W. Ryu, T.-Y. Kwon, M. Choi, and C.-M. Kim, J. Phys. A: Math. Theor. 41, 275102 (2008).

[48] E. Krioukov, D. J. W. Klunder, A. Driessen, J. Greve, and C. Otto, Opt. Lett. 27, 512 (2002).

[49] Q. Zhong, J. Ren, M. Khajavikhan, D. N. Christodoulides, Ş. K. Özdemir, and R. El-Ganainy, Phys. Rev. Lett. 122, 153902 (2019). 
[50] L. Ge, D. Liu, A. Cerjan, S. Rotter, H. Cao, S. G. Johnson, H. E. Tureci, and A. D. Stone, Opt. Express 24, 41 (2016).

[51] M. Sargent III, M. O. Scully, and W. E. Lamb, Jr., Laser Physics (Addison-Wesley, Reading, MA, 1974).

[52] M. Sargent III, Phys. Rev. A 48, 717 (1993).

[53] W. J. Chen, J. Zhang, B. Peng, S. K. Ozdemir, X. D. Fan, and L. Yang, Photon. Res. 6, A23 (2018).
[54] B. Peng, S. K. Ozdemir, F. C. Lei, F. Monifi, M. Gianfreda, G. L. Long, S. H. Fan, F. Nori, C. M. Bender, and L. Yang, Nat. Phys. 10, 394 (2014).

[55] J. Wiersig, Phys. Rev. A 93, 033809 (2016).

[56] J. G. Zhu, S. K. Ozdemir, Y.-F. Xiao, L. Li, L. N. He, D.-R. Chen, and L. Yang, Nat. Photonics 4, 46 (2009). 